\title{
Highly nutrient-dense spreads: a new approach to delivering multiple micronutrients to high-risk groups
}

\author{
André Briend \\ Institut de Recherche pour le Développement, ISTNA-CNAM, 5 rue du Vert Bois, 75003 Paris, France
}

\begin{abstract}
Using a highly fortified food is the most attractive option to bringing missing nutrients to vulnerable groups. The recent development of a highly nutrient-dense spread (HNDS) for the treatment of malnourished children may have some relevance for other high-risk groups. Traditionally, severely malnourished children are fed for 3-4 weeks during their recovery with adapted milk feeds prepared by mixing dried skimmed milk, oil and sugar with a vitamin and mineral complex. This approach, however, is difficult to implement, since these feeds are excellent growth media for bacteria, and they must be prepared and fed under close supervision. This constraint led to the development of a HNDS, which is obtained by replacing part of the dried skimmed milk with a mixture of groundnut butter and powdered lactoserum. This spread can be eaten without dilution with water and preliminary trials showed that children preferred this HNDS to traditional liquid diets. In HNDS all powdered ingredients are embedded in fat which protects vitamins against oxidation and increases the shelf life of this product. Spreads also have a very low humidity and bacteria do not grow in it. Attempts to use spreads to supplement other vulnerable groups such as moderately malnourished children and pregnant women are discussed.
\end{abstract}

Fat spreads: Nutrient dense food: Dietary supplements

Diets prepared from low-cost local foods in developing countries often have a low vitamin and mineral content. Moreover, they often have a high phytate content, which prevents absorption of some key nutrients, such as calcium, zinc or iron (Ferguson et al. 1988). Staple food fortification is usually the cheapest approach to increase the mineral and vitamin intake of the whole population since all missing micronutrients are usually much cheaper in chemical form than when purchased in locally available foods (Bauerfeind \& Lachance, 1991). Yet, fortification of a staple food is not well adapted for delivering micronutrients to high-risk groups: some of them need a high level of fortification which would not be suitable for the general population or would be too expensive to implement on a large scale.

Diets used for the treatment of severe malnutrition represent an extreme case, since malnourished patients have very high vitamin and mineral requirements, and no combination of natural foods is rich enough to fully cover their nutritional needs. Hence, modern treatment of severe malnutrition relies on the use of heavily fortified milk feeds. Recently, a highly nutrient-dense spread (HNDS) has been proposed to feed malnourished children during the recovery phase for moderate cases of malnutrition (Briend et al. 1999). This new approach may have some relevance for programmes delivering micronutrient supplements to other vulnerable groups.

\section{Recent developments in the management of severe malnutrition}

According to the most recent World Health Organization (WHO) recommendations, severely malnourished children receive, during the rehabilitation phase, large quantities of high energy high protein milk feeds with added vitamins and minerals as the only food (WHO F100) to promote rapid weight gain (World Health Organization, 1999). This feeding scheme, however, is difficult to implement: the milk feeds, obtained by mixing together dried skimmed milk, oil and sugar with a vitamin-mineral mix are prepared by dilution with water and are excellent growth media for bacteria. These feeds can be safely prepared and used only under close supervision in centres with access to clean water. This constraint seriously limits the coverage of nutritional rehabilitation programmes based on WHO recommendations. To avoid this problem, a highly nutrientdense spread, has recently been developed with a nutritional value close to WHO F100, which can be directly eaten by the child, without prior addition of water. This could be obtained by replacing part of the dried skimmed milk of the F100 formula by powdered lactoserum mixed with peanut butter, and oil by a vegetable fat mixture including essential fatty acids and with an adequate viscosity and melting point (Briend et al. 1999). These 
ingredients are mixed together to obtain a spread into which all other components are included. The resulting product looks and tastes like peanut butter. It has a very low water content, since two of its main components, peanut butter and vegetable fat do not contain any water. For the same reasons, it is not water-soluble. These properties seem to be of a major advantage when preparing highly fortified foods for vulnerable groups.

\section{Properties of spreads compared to liquid products}

\section{Increased acceptability}

A first acceptability trial of HNDS designed for the treatment of severe malnutrition compared to liquid WHO F100 showed that a spread is clearly preferred by children. This first study took place in a therapeutic feeding centre in Chad where children received six WHO F100 feeds per day. To test the acceptability of the spread, half of the F100 feeds were replaced by HNDS, and energy intake per feed was compared. The order of the feed changed everyday to avoid a confounding effect of the time of the feeds. These malnourished children took on average $168.4 \pm 70.0 \mathrm{~kJ} / \mathrm{kg}$ per feed (SD) of the HNDS compared to $84.4 \pm 38.4 \mathrm{~kJ} / \mathrm{kg}$ per feed of the liquid WHO F100 $(P<0.001)$ (Fig. 1) (Briend et al. 1999).

The high energy intake observed with the spread may partly be explained by its very high energy density: the spread contains $2280 \mathrm{~kJ} / 100 \mathrm{~g}$ compared to $418 \mathrm{~kJ} / 100 \mathrm{~g}$ for liquid WHO F100 and children are known to have a clear preference for high energy density foods (Birch et al. 1990). This factor may have offset the effect of viscosity: when isocaloric meals are given to children, intake is higher for lower viscosity feeds (Bennett et al. 1999) and the high viscosity of the spread is likely to have limited the increased energy intake compared to the liquid F100.

A recent study showed that energy intake in adult women volunteers was higher from a meal where water and food were given separately compared with the same meal mixed with water (Rolls, Bell \& Thorwart, 1999). The increased acceptability of the spread compared to its liquid equivalent would suggest that the same phenomenon may occur in malnourished children, and that the traditional liquid feeds

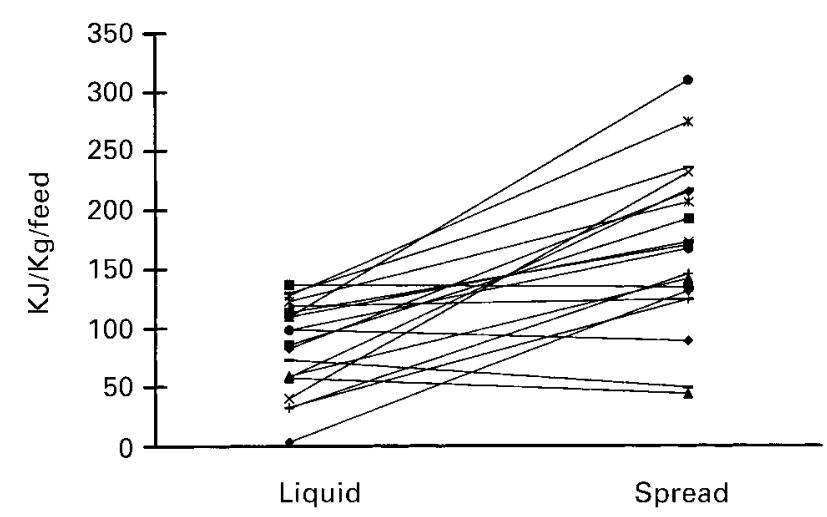

Fig. 1. Comparison of energy intake with liquid WHO F100 and a spread with similar nutritional characteristics (Briend et al. 1999) (C) The Lancet Ltd, 1999. may limit energy intake during the recovery compared to a HNDS given separately from water. This could also explain part of increased energy intake observed with the HNDS compared to a liquid feed.

Independent of its high energy density, the spread developed for the treatment of severe malnutrition may have a better taste than that of the liquid version; fat undoubtedly masks the unpleasant taste of some minerals and vitamins present in high concentrations and in soluble form in these formulae and this is well perceived in the traditional liquid diets. Yet, malnourished children are likely to have a different taste perception, as a result of their young age and frequently associated zinc deficiency and it is not sure that this taste factor is really important. Taste, however, may be important, for other vulnerable groups, such as pregnant women or elderly patients.

\section{Protection against oxidation}

A spread has limited contact with oxygen and humidity from the atmosphere through its external surface in contrast to flour, or any food made of small particles such as powdered milk. This difference is explained by the decreasing surface to volume ratio for larger particles. It can be shown that this ratio is related to the diameter ratio and that for instance, the surface of contact with atmosphere of a sphere with a $30 \mathrm{~mm}$ diameter is 100 times smaller than the same weight made of 1000000 spheres of $0.3 \mathrm{~mm}$ diameter.

The physical structure of a spread also protects it against oxidation. A spread can be regarded as an emulsion of powder mixed into fat. This is in contrast with full cream powdered milk, which must be water soluble, and which is a fat into powder type of emulsion. The type of emulsion (powder into fat) used in spreads is an advantage when incorporating vitamins, because this thick fat layer around them protects them against humidity and oxygen.

Spreads contain only very small quantities of water, usually less than $2 \%$ compared to $8-12 \%$ for flour. This property is related to the fat ingredients used to prepare a spread, which do not contain any water and do not attract humidity from the atmosphere. The absence of water also prevents soluble minerals from reacting with vitamins and helps in increasing their shelf life.

The limited contact surface with the atmosphere, the protection of vitamins and minerals by a thick fat layer, and absence of water are all very favourable factors for obtaining a prolonged shelf life for a fortified spread by increasing its resistance to oxidation.

\section{Resistance of spreads to bacteria and insects}

Bacteria need water to grow. The water content of a ready to use HNDS is always lower than that of a flour before cooking and of course much lower than for a traditional porridge after cooking or for a milk feed diluted with water, both with at least $80 \%$ humidity. Bacteria introduced experimentally into a HNDS do not grow, whereas they grow exponentially for several hours in the liquid version of the same feed. Fig. 2 shows bacterial growth at $40^{\circ} \mathrm{C}$ of E. coli ATCC 8739 experimentally introduced in $\mathrm{WHO}$ 


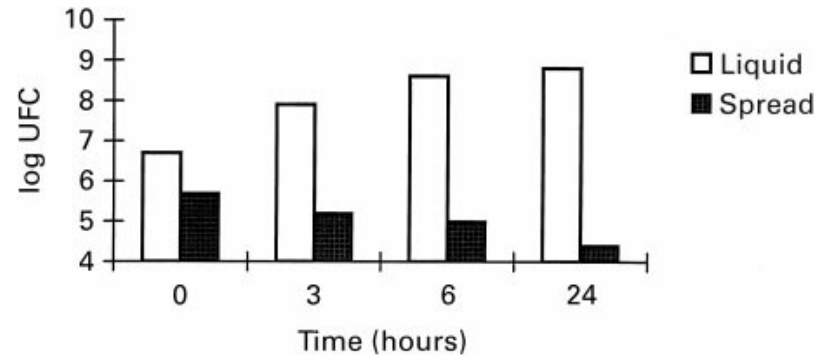

Fig. 2. Comparison of bacterial growth in liquid and solid rehabilitation diets (log scale).

F100 formula after addition of water and in its ready to use HNDS version. If taken like a snack by itself or with bread or a biscuit, a spread is safe because like bread or biscuits, it does not contain enough water for bacteria to grow. This makes the association between local bread and a spread a very safe option for supplementary feeding programmes in bread eating communities.

Most insects found in flour bags take the water they need for their growth and metabolism from the humidity present in the flour. Flours are notoriously difficult to protect against insects in tropical climates with high air humidity (Walker, 1992). Spreads do not contain enough water to support insect growth, and for this reason are much easier to store than flours.

\section{Development of a spread for supplementing the diet of moderately malnourished children}

Design of a spread for supplementary feeding programmes

The excellent acceptability of the spread developed to treat severe malnutrition led to the development of a second

Table 1. Comparison of a spread used for recovery of severe malnutrition (WHO F100 copy) and of a highly nutrient dense (HNDS) spread used to reverse stunting and given in addition to the local diet

\begin{tabular}{llcc}
\hline & & F 100 copy & HNDS \\
\hline Energy & $\mathrm{kJ}$ & 2268 & 2628 \\
Proteins & $\mathrm{g}$ & 13.5 & $10 \cdot 2$ \\
Lipids & $\mathrm{g}$ & $35 \cdot 3$ & $57 \cdot 05$ \\
Calcium & $\mathrm{mg}$ & 320 & 1000 \\
Phosphorus & $\mathrm{mg}$ & 394 & 628 \\
Potassium & $\mathrm{mg}$ & 1111 & 1135 \\
Magnesium & $\mathrm{mg}$ & 92 & 156 \\
Iron & $\mathrm{mg}$ & 11.5 & 42 \\
Zinc & $\mathrm{mg}$ & 14 & 41 \\
Copper & $\mathrm{mg}$ & 1.78 & 2 \\
& & & \\
Vitamins & & & \\
A & $\mu \mathrm{g}$ & 910 & 2000 \\
$\mathrm{D}$ & $\mu \mathrm{g}$ & & 50 \\
$\mathrm{E}$ & $\mathrm{mg}$ & 20 & 20 \\
$\mathrm{C}$ & $\mathrm{mg}$ & 53 & 125 \\
$\mathrm{~B}_{1}$ & $\mathrm{mg}$ & 0.6 & 3.5 \\
$\mathrm{~B}_{2}$ & $\mathrm{mg}$ & 1.8 & 4 \\
$\mathrm{~B}_{6}$ & $\mathrm{mg}$ & 0.6 & 3.5 \\
$\mathrm{~B}_{12}$ & $\mu \mathrm{g}$ & 0.53 & 3.5 \\
Folic acid & $\mu \mathrm{g}$ & 210 & 500 \\
Pantothenic acid & $\mathrm{mg}$ & 3.1 & 25 \\
Niacin & $\mathrm{mg}$ & 5.3 & 50 \\
\hline
\end{tabular}

HNDS which could be used to supplement local diets, and make them suitable for the management of moderate malnutrition. This product is different from the copy of the WHO F100 tested in the above quoted study from Chad: WHO F100 is designed to be used as the only food during nutritional rehabilitation and its fortification levels with vitamins and minerals are calculated to cover all the nutritional requirements of a malnourished child consuming between 420 and $840 \mathrm{~kJ} / \mathrm{kg}$ from this product. This makes the use of F100 derived products expensive compared to nutritional rehabilitation diets prepared with locally available foods. This second spread was developed to provide in a small volume the minerals and vitamins missing in a rehabilitation diet prepared with locally available foods.

\section{Adjusting mineral and vitamin concentrations}

All minerals and vitamins needed to balance a wheat-based diet were added to $50 \mathrm{~g}$ of fat spread, without decreasing its acceptability. Spreads make possible the use of virtually tasteless insoluble mineral salts for fortification since their viscosity prevents sedimentation of minerals during storage. Moderately malnourished children usually have an adequate gastric acidity and absorption of these minerals should be possible. Very high fortification levels with insoluble salts can be obtained without adverse effect on acceptability. Fortification levels chosen for this second spread were derived from recommendations of a meeting on supplementary feeding programmes (Golden et al. 1995). Fortifications levels reached for the spread designed for supplementary feeding programmes are higher than for the HNDS copy of WHO F100 (Table 1). Since not more than $55 \%$ of energy should come from fat, a limit can be set for the maximum quantity of each nutrient included in this fat supplement to remain within the range of a safe intake level.

Groundnuts have lysine as limiting amino acids, and it may be advantageous to add some soy flour to improve the amino acid profile of the spread if prepared with groundnuts. Spreads prepared with groundnuts contain phytates that may be further increased by addition of soy flour. Yet, fortification levels of this food for zinc are high enough to keep phytate:zinc and phytate:iron ratios within acceptable limits. The spread designed for supplementing malnourished children had a phytate to zinc ratio of $2: 2$ despite inclusion of groundnuts and of soy flour.

Using a spread for supplementary feeding programmes is at odds with the design of most ongoing programmes, based on the use of high protein low fat foods made from blended flours. Composition of the HNDS is clearly different from the most commonly used blended foods (Hertz, 1997) (Table 2). The high protein content of most currently used blended flours may be related to a design going back to the days when protein deficiency was thought to be the most common nutritional problem in developing countries and little attention was given to mineral fortification (Anonymous, 1999). In any case, a fat supplement with a high vitamin and mineral content seems better adapted for supplementary feeding than high protein 
Table 2. Comparison of the macro-nutrient content (in \% weight) of the HNDS and of blended flours usually given in supplementary feeding programmes

\begin{tabular}{lccc}
\hline & Proteins & Carbohydrates & Lipids \\
\hline Blended foods & 14 & 70 & 7 \\
HNDS & 11 & 18 & 57
\end{tabular}

foods since low cost diets usually have a low fat low mineral and vitamin content.

A first efficacy trial of HNDS to feed moderately malnourished children has been recently carried out in Algeria in a group of stunted anaemic children (Branca et al. 1999). The intervention group was compared to a control group receiving a non-fortified spread. After 3 months of supplementation, the haemoglobin level of these children increased from a mean level of $9.2 \pm 2.3$ to $11.8 \pm 1.5 \mathrm{~g} / 100 \mathrm{ml}(P<0.001)$ and a significant reduction of $6 \%$ of the prevalence of stunting was observed $(P<0.05)$. This efficacy is in contrast with lack of consistent results observed when evaluating supplementation feeding programmes based on high protein blended flours (Beaton \& Ghassemi, 1982).

\section{Using a spread for other supplementary feeding programmes}

The advantages of a fortified spread can be extended to other programmes attempting to deliver micronutrient supplements to high-risk groups. Development of food for supplementing pregnant and lactating women is another possible application and is now under way. High intakes of easily absorbable iron are usually required to maintain iron stores in pregnant women living in developing countries and usually consuming a diet with a low content of easily absorbable haeme iron. Iron requirements for women who are already anaemic at the beginning of pregnancy may reach $60 \mathrm{mg}$ per day, or more, which can in practice only be achieved by giving iron tablets. Iron supplementation programmes, however, are usually impaired by acceptability problems due to the bad taste and secondary effects of these tablets, particularly when iron is given in soluble form. In fact, it is possible to get around the taste problem by adding $60 \mathrm{mg}$ of iron to $50 \mathrm{~g}$ of spread, without markedly affecting its taste. Field trials are now in progress to see whether unpleasant secondary effects are also reduced when iron is put into HNDS. The efficacy of this food-based approach is also being compared with the more traditional form of iron supplementation with iron sulphate tablets.

A highly dense nutrient spread could also be developed for other applications, such as supplementation of elderly patients with limited chewing capacity, or supplementation of hospital patients.

\section{Flexibility of spread formulation}

The formulation of the HNDS can easily be adapted to different situations. Firstly, the ingredients can be changed to incorporate locally available products or to respond to specific nutritional needs. Groundnuts can be replaced by soy or other proteins and fat rich foods in places where allergy to peanuts may be a problem, or where other ingredients are more readily available. Cocoa, or hazelnuts, or any locally available fat dried fruit can replace them. Essential fatty acid content of the spread can be adjusted, if needed.

A spread can also be eaten along with other foods, or added to a traditional dish as a condiment, if appropriate flavours are added. Spreads usually have a low viscosity above $37^{\circ} \mathrm{C}$ and quickly melt away when added to a dish.

The fortification level of each nutrient can be also adapted to different nutrition programmes and to specific settings according to the most prevalent nutritional deficiency. The quantity of fat supplement added may be reduced if nutrients to be added have high safe upper limits of intake, thus decreasing the cost.

\section{Sustainability of spread based programmes}

The technology to prepare spreads is simple and can be easily implemented in most developing countries. For the reasons explained above, fortification of these spread should be less difficult than for blended flours, the currently favoured food for supplementation programmes of vulnerable groups. Non-fortified spreads are already produced and sold to large segments of the population in developing countries. This suggests that production of fortified spreads should be economically sustainable.

\section{Conclusions}

This flexible HNDS technology opens new perspectives for multiple nutrient supplementation programmes targeted at different high-risk groups. Clearly, field studies are desired to evaluate the feasibility of this new approach compared to other traditional supplementation programmes.

\section{References}

Anonymous (1999) Enhancing the nutritional quality of relief diets. In Workshop Proceedings Food Aid Management. Washington DC 28-30 April.

Bauerfeind JC \& Lachance PA (1991) Nutrition Addition to Food - Nutritional, Technological and Regulatory Aspects. Trumbull: Food \& Nutrition Press.

Beaton GH \& Ghassemi H (1982) Supplementary feeding programs for young children in developing countries. American Journal of Clinical Nutrition 35 (4 Suppl), 863-916.

Bennett VA, Morales E, Gonzalez J, Peerson JM, Lopez de Romana GL \& Brown K (1999) Effects of viscosity and energy on total daily energy consumption by young Peruvian children. American Journal of Clinical Nutrition 70, 285-291.

Birch LL, McPhee L, Steinberg L \& Sullivan S (1990) Conditioned flavor preferences in young children. Physiology and Behavior 47, 501-505.

Branca F, Lopriore C, Guidoum Y, Briend A \& Golden MH (1999) Multi-micronutrient fortified food reverses growth failure and anaemia in 2-5-year-old stunted refugee children. Scandinavian Journal of Nutrition 43, 51S.

Briend A, Lascala R, Prudhon C, Mounier B, Grellety Y \& Golden MHN (1999) Ready to use therapeutic food for treatment of marasmus. Lancet 353, 1767-1768.

Ferguson EL, Gibson RS, Thompson LU, Ounpuu S \& Berry M (1988) Phytate, zinc, and calcium contents of 30 East African 
foods and their calculated phytate: Zn, Ca:Phytate, and $[\mathrm{Ca}][$ Phytate]/[Zn] molar ratios. Journal of Food Composition and Analysis 1, 316-325.

Golden M, Briend A \& Grellety Y (1995) Report of a meeting on supplementary feeding with particular reference to refugee populations. European Journal of Clinical Nutrition 49, 137145.

Hertz G (1997) Production of pre-cooked fortified blended foods in Kenya: a success story. Field Exchange 2, 6-7.
Rolls BJ, Bell EA \& Thorwart ML (1999) Water incorporated into a food but not served with a food decreases energy intake in lean women. American Journal of Clinical Nutrition 70, 448455.

Walker D (Editor) (1992) Food storage manual. Chatham, National Resources Institute \& Rome, World Food Programme. World Health Organization (1999) Management of severe malnutrition: a manual for physicians and other senior health workers. Geneva: World Health Organization. 